\title{
ISTEC-MRS Workshop Covers Processing of High-Temperature Superconductors
}

An international workshop on Controlled Processing of High-Temperature Superconductors: Fundamentals and Applications was held at the Maui InterContinental Resort, Hawaii, on June 17-21, 1995. The workshop was co-sponsored by the International Superconductivity Technology Center (ISTEC) of Japan and the Materials Research Society. From 1989 through 1991, and in 1993 and 1994, ISTEC sponsored workshops in Japan on various topics in superconductivity. In 1992, MRS co-sponsored with ISTEC a workshop in Honolulu, Hawaii, on Controlled Crystal Growth of High-Temperature Superconductors. This year's workshop was a direct descendant of the previous workshops and embraced a subject similar to that of the previous Hawaii gathering. As such, progress made over the past three years in processing and properties could be readily assessed by the talks and posters presented.

The goals of the 1995 Workshop were implicit in the session topics: controlled crystal growth, strong flux pinning, process control for thin film growth, bulk applications, thin film applications, transport and current paths, and processing and characterization of wires and tapes. The combination of state-of-the-art information and focused audience interest, present within an informal and pleasant atmosphere, was thought to be ideal for promoting exchange among researchers, and thus for furthering the drive toward full-scale emergence of superconducting technologies.

The workshop was chaired by Shoji Tanaka of ISTEC and Julia M. Phillips of MRS. Financial support was provided by ISTEC, the Japan Keirin Association, the International Science Foundation, the National High Magnetic Field Laboratory at Florida State University, the Texas Center for Superconductivity at the University of Houston, the Science and Technology Center for Superconductivity at the University of Illinois at UrbanaChampaign, and the DOE-funded Superconductivity Technology Centers at Argonne, Oak Ridge, and Los Alamos National Laboratories. The four-day program was attended by 181 people from 16 countries. In addition to three days of talks, approximately 100 posters were presented.

The technical program began with an Opening Ceremony, followed immediately by a series of plenary talks. C.W. Chu (University of Houston) talked on new materials, emphasizing work with $\mathrm{Hg}$ based materials, and compounds such as $\left(\mathrm{Cu}_{1-x} \mathrm{C}_{x}\right) \mathrm{Ba}_{2} \mathrm{Ca}_{n-1} \mathrm{Cu}_{n} \mathrm{O}_{y}, \mathrm{Sr}_{2} \mathrm{RuO}_{4}$, and
$\mathrm{Sr}_{2} \mathrm{CuO}_{4}$. Fundamental issues related to $T_{c}$ and doping were addressed. $M$. Murakami (ISTEC) followed with a talk on melt-processed RE-123 materials. Apparently several applications will require improvements in flux pinning over that of conventional Y-123. Murakami summarized recent improvements obtained by doping, including use of Nd-123. D. Larbalestier (University of Wisconsin) talked about another material envisioned for widescale use, clad $\mathrm{Bi}-2223$ tapes. The focus was on transport paths and microstructural features that limit critical current density. Y. Tanaka (Furakawa Electric) spoke on power applications of superconductors. Significant progress has been made toward commercialization of current leads, transmission cables, and coils based on hightemperature superconductor (HTS) technology. The presentations then shifted to electronic applications. H. Hayakawa (Nagoya University) discussed the present status of HTS grain-boundary and ramp-edge junctions. He concluded that improvements in the products of $\mathrm{I}_{c} \times \mathrm{R}_{n}$ product uniformity, and reproducibility are required before significant commercialization can occur. Paul Müller (Walther-Meissner) concluded the Plenary Session with a discussion of intrinsic Josephson effects in Bi- and Tlbased film junctions and their possible application as the high-power emitter for $\mathrm{GHz}$ microwaves.

Sessions on Controlled Crystal Growth and Strong Flux Pinning followed. The first five crystal-growth talks were devoted to Bi-Sr-Ca-Cu-O materials. Over the past few years, significant advances have been made in production of phase-pure materials. Recent work on control of composition to produce whiskers or increases in flux pinning was summarized. Use of pure oxygen during processing was also discussed. As much for convenience as anything, air was used in most of the early work with Bi-Sr-Ca-Cu-O. Advantages of oxygen include avoidance of certain deleterious second phases and minimization of problems associated with carbon. Three talks on Y-123 growth focused on fundamental issues and techniques devoted to directional growth. Remarkable progress has been made, and high-quality single crystals of $Y B C O$ can now be made $\approx 17 \times$ $17 \times 12 \mathrm{~mm}$. The final talk in the session focused on growth under hyperbaric conditions of $\mathrm{Hg}$-based and infinite-layer single crystals. These crystals are proving to be highly useful in fundamental studies of superconducting properties.
The Strong Flux Pinning Session was dominated by recent results in the $\mathrm{Y}-123$ system. Effective pinning centers have been introduced by heavy-ion irradiation, fission-induced damage, and controlled precipitation. The discussion on pinning centers in $\mathrm{Bi}-2212$ and $\mathrm{Bi}-2223$ included the role of oxygen point defects and incorporation of carbon nanotubes. Two talks dealt with fundamental issues related to flux pinning, in particular, how limited researchers' current understanding of mechanisms is and how much more work is required. The subsequent session, on bulk applications, comprised a series of talks on uses for melt-processed Y-123 and its alloys. Although full fundamental understanding of the materials has yet to emerge, substantial progress has been made toward implementation of flywheels, bearings, motors, and magnets. For most of these applications, current materials performance is more than adequate. Challenges have shifted to ensuring reproducibility at a low cost. This point was emphasized when a questioner informed the audience that nonsuperconducting technologies can be strong competitors to superconductors. For example, gas turbines are an inexpensive alternative to flywheels in many possible utilityrelated applications. In the ensuing discussion, the speakers were quick to point out that utility involvement in flywheel research ensures that economic factors are being respected.

Sessions on thin-film growth and applications covered wide ranges of materials and approaches. Growth of Y-123, Bi2212, Bi-2223, and Tl-based films by techniques including metal-organic chemical vapor deposition (MOCVD), electron beam deposition, molecular beam epitaxy, plasma deposition, and RF sputtering was discussed. Substrates being used include $\mathrm{MgO}, \mathrm{SrTiO}_{3}, \mathrm{LaAlO}_{3}, \mathrm{NdGaO}_{3}$, and $\mathrm{CeO}_{2}$, and $\mathrm{ZrO}_{2}$ buffer layers on sapphire or Ni-based alloys. Significant progress was reported on obtaining the smooth surfaces that are imperative for device fabrication.

Thin-film applications were primarily of three types. Near-term goals were production of coated $Y-123$ wires for current transport, and $\mathrm{Y}-123$ films for microwave and band-pass filters, resonators, and delay lines. Long-term goals included production of junction devices, primarily from $Y-123$.

Two sessions were devoted to bulk conductors. The first session focused on issues related to transport current paths. The talks were evenly divided among Y- 
123, Bi-based materials, and Tl-based materials. Issues covered included grainboundary structure, grain connectivity, texture, and percolation paths. For $\mathrm{Y}-123$, very high $J_{c}$ values $>10^{6} \mathrm{~A} / \mathrm{cm}^{2}$ at $77 \mathrm{~K}$ are now reported for biaxially textured tapes. Texture of the $c$ axis alone appears to limit $J_{c}$ at $77 \mathrm{~K}$ to $\approx 10^{4} \mathrm{~A} / \mathrm{cm}^{2}$. For $\mathrm{Bi}$ 2212 and $\mathrm{Bi}-2223$, evidence was presented for transport current being limited by the connectivity between $a-b$ planes of each grain. Percolation of such connections in powder-in-tube tapes appears to be possible. Tl-1212 and Tl-1223 tapes prepared by conventional powder-in-tube techniques and vapor-deposition processes were discussed. $J_{c}$ values in field remain low for the former technique. An interesting recent development in tapes made by the latter technique is use of textured $\mathrm{Ag}$ substrates. Combinations of warm working and recrystallization can produce strong $\{100\}\langle 001\rangle$ textures in the Ag. Biaxial alignment of Tl-1223 on such Ag may, therefore, be possible. A few groups are now working on this process; to date, $j$ values have remained relatively low. However, Tl-1223 deposits on Ag have exhibited transport $J_{c}$ values close to $10^{5} \mathrm{~A} / \mathrm{cm}^{2}$ at $77 \mathrm{~K}$, and thus the prospects for success with this technique are good.

Bi-based materials have made the most progress toward bulk conductor applications. Bi-2212 and Bi-2223 current leads are now available. Significant improvements have been made to transmission cables, busbars, solenoids, and magnets. For Bi-2212, many applications are envisioned for $4.2 \mathrm{~K}$. Bi-2223 appears to be suitable for high-field applications at temperatures less than about $40 \mathrm{~K}$, or at higher temperatures in lower fields. Because of improvements in wire and tape manufacture, react-and-wind processing now competes with wind-and-react methods.

In keeping with the spirit of identifying and assessing progress made over the past three years, the Workshop featured an Awards Ceremony for superior performance in materials or devices. Recipients were Y. Shiohara, S. Yao, Y. Hamikawa, H. Zama, and S. Koyama of the Superconductivity Research Laboratory of ISTEC for several developments in quality and size of $\mathrm{Y}-123$ single crystals; W-K. Chu and colleagues of the University of Houston for fabrication of a $19-\mathrm{kg}$ flywheel and a vacuum bearing operating at $80 \mathrm{~K}$ for lunar applications; $\mathrm{Y}$. Tanaka and his colleagues of Furakawa Electric for producing 5-m, multifilament $66 \mathrm{kV}$ cables capable of carrying 2,000 A; O. Kohno and colleagues from Fujikura Ltd. for producing high $-J_{c}$, flexible $Y-123$

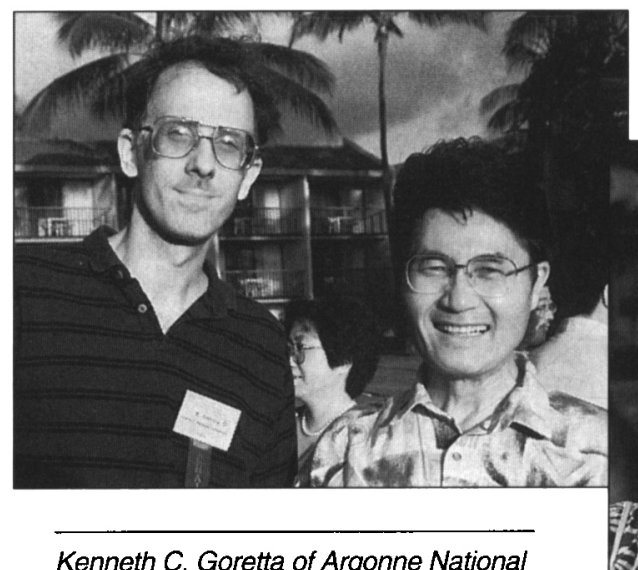

Kenneth C. Goretta of Argonne National Laboratory (MRS) (left) and Koichi Kitazawa of the University of Tokyo (ISTEC) chaired the joint program committee.

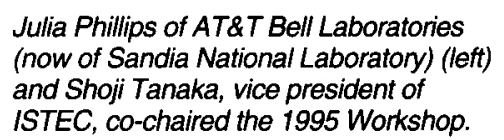

conductors; a group from American Superconductor for a wound magnet of Ag-clad Bi-2223 producing $0.61 \mathrm{~T}$ at $77 \mathrm{~K}$; $K$. Sato and colleagues from Sumitomo Electric for several accomplishments with cables and wound magnets, including producing a $3 \mathrm{~T}$ field at $20 \mathrm{~K}$; R. Weinstein and colleagues from the University of Houston for a trapped-field magnet (2.6 T at $77 \mathrm{~K}, 8.34 \mathrm{~T}$ at $54 \mathrm{~K}$ ); W. Gawalek and colleagues from the Institut für Physikalische Hochtechnologie, Jena, for a high-power-density hysteresis motor; $\mathrm{S}$. Oda and colleagues from Tokyo Institute of Technology for extremely smooth thin films made by MOCVD; $\mathrm{H}$. Kinder and colleagues from the Technical University of München for 25-cm-diameter high-quality $\mathrm{Y}-123$ thin films with $J_{c}$ values $>10^{6} \mathrm{~A} / \mathrm{cm}^{2}$ at $77 \mathrm{~K}$; and $\mathrm{N}$. Chew and colleagues of DRA Malvern for fabrication of a multilayer flip chip gradiometer with superior performance.

Each of the awards represents an accomplishment that brings HTS technology close to commercialization. For example, a field of $0.61 \mathrm{~T}$ had been thought to be impossible to produce from Bi-2223. Not only was it achieved, but this field is sufficient for applications such as magnetic resonance imaging. A $3 \mathrm{~T}$ field at $20 \mathrm{~K}$ is also highly significant because such a temperature can be easily obtained with inexpensive, reliable cryocoolers. Wires longer than those now produced will, however, be required for most future magnet applications. Now that react-and-wind technology is available, production of larger magnets should be feasible. Trapped-field magnets mirror the successes of the wound magnets. A field of $2.6 \mathrm{~T}$ at $77 \mathrm{~K}$ is sufficient for applications such as maglev trains.

Film technology has advanced significantly. Layer-by-layer control, film smoothness, and superconducting properties are excellent. The existence of a $25-\mathrm{cm} Y-123 \mathrm{film}$ with a $J_{c}>10^{6} \mathrm{~A} / \mathrm{cm}^{2}$ at $77 \mathrm{~K}$ was a surprise to many of the workshop attendees. In combination with the multilayer fabrication technology of the gradiometer and intrinsic Josephson devices, film technology appears to be entering the early stage of practical applications.

Nearly a decade has passed since the advent of high-temperature superconductivity. Discovery of new materials and development of fundamental insight has occurred and continues to occur frequently, if sporadically. In contrast, the progress toward commercialization of the technology has been steadily accelerating. Products such as current leads, sensors, levitators, and coils are now for sale. Filters, transmission cables, and larger magnets are likely to follow soon. In many cases, the next stage of development is centered on cost versus performance rather than property enhancement. The consensus of the workshop attendees was that most larger-scale applications will require more years to realize, however, and that consistent technological advancement will be required in the interim to maintain levels of funding and interest.

KENNETH C. GORETTA AND KOICHI KITAZAWA 